\title{
Erratum to: Studies on abacavir-induced hypersensitivity reaction: a successful example of translation of pharmacogenetics to personalized medicine
}

\author{
GUO YongLi ${ }^{1}$, SHI LeMing ${ }^{2}$, HONG HuiXiao ${ }^{2}$, SU ZhenQiang ${ }^{2}$, \\ FUSCOE James $^{1} \&$ NING BaiTang ${ }^{1}$ \\ ${ }^{1}$ Division of Systems Biology, National Center for Toxicological Research, the US Food and Drug Administration, Jefferson, AR 72079, USA; \\ ${ }^{2}$ Division of Bioinformatics and Biostatistics, National Center for Toxicological Research, the US Food and Drug Administration, \\ Jefferson, AR 72079, USA \\ Erratum to: SCIENCE CHINA Life Sciences, February 2013 Vol.56 No.2: 119-124 \\ doi: 10.1007/s11427-013-4438-8
}

The following statement should be included in the manuscript:

The views presented in this article do not necessarily reflect those of the US Food and Drug Administration.

The online version of the original article can be found at http://dx.doi.org/10.1007/s11427-013-4438-8 